\title{
Accurate Iris Boundary Detection in Iris-Based Biometric Authentication Process
}

\author{
Somnath Dey and Debasis Samanta \\ School of Information Technology, \\ Indian Institute of Technology, Kharagpur, India-721302 \\ somnath_dey2003@yahoo.co.in, dsamanta@iitkgp.ac.in
}

\begin{abstract}
This paper presents an efficient technique for accurate detection of iris boundary, which is an important issue for any iris-based biometric identification system. Our proposed technique follows scaling, histogram equalization, edge detection and finally removal of unnecessary edges present in the eye image. Scaling and removing unnecessary edges enables us to reduce the search space for iris boundary. Experimental results show that with our approach it is possible to detect iris boundary as much as $98 \%$ of the eye images in CASIA database accurately and it needs only $25 \%$ time compared to the existing approaches.
\end{abstract}

Keywords: Iris recognition; biometric authentication; image segmentation; image processing.

\section{Introduction}

Now a days, biometric system is widely used for automatic recognition of an individual based on some unique characteristic owned by the individual. Iris recognition is a method of biometric identification based on high-resolution images of the irises of an individual's eyes. Iris is a thin contractile diaphragm, which lies between pupil and the white sclera of a human eye. Different parts of an eye image is shown in Fig. 1.

In iris biometric system, an important task is iris localization. It is observed that the iris localization task is the most computationally intensive task. In iris localization task, we try to locate iris part from an eye image. Iris part

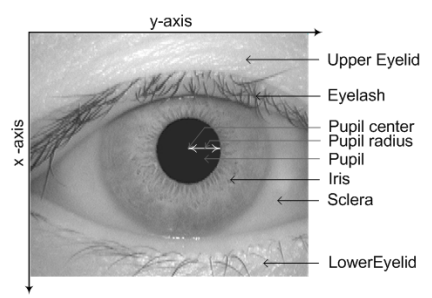

Fig. 1. The typical components in an eye image

A. Ghosh, R.K. De, and S.K. Pal (Eds.): PReMI 2007, LNCS 4815, pp. 600607, 2007.

(C) Springer-Verlag Berlin Heidelberg 2007 
localization is necessary to isolate the iris part of the image in between the iris boundary (between sclera and iris) and outside the pupil. This task mainly consists of two sub tasks: detecting pupil boundary (between pupil and iris), and detecting iris boundary (between iris and sclera).

Efficiency of iris recognition in any iris based biometric authentication process greatly relies on how much accurately we detect the iris part from an eye image. The standard procedure to detect an iris boundary is to detect pupil center, the pupil radius, and subsequently use these information to find out iris boundary. Even though a successful detection of pupil helps us to limit the search space significantly, still an issue remains. The pupil and iris center are not necessarily concentric. We may trap in an improper outcome if the issue as stated above is not taken into consideration. Therefore, finding the coordinate of the iris center and radius are must.

In this paper, we detect the pupil boundary and capture pupil information (that is, pupil radius and pupil center) initially. This pupil information is used to detect iris center and radius followed by detecting the iris boundary. We propose an efficient approach to achieve the above mentioned tasks. In addition to these, we also address other issues such as, low contrast between sclera and iris, non uniform illumination, noise due to eye lashes, iris region obscured by eyelids and eye lashes, white spot due to reflection of light etc.

\section{Related Work}

Daugman's system [12] uses integro-differential operator to detect pupil and iris boundary. This operator is sensible to the specular spot reflection of non diffused artificial light. Wildes [3] uses binary edge map and voting each edge points to instantiate particular contour parameter values to detect pupil and iris boundary. Li Ma et al. 4 calculate the summation of intensity value along each row and each column. They choose the particular row and column along which summations are minimum. That row and column are used as approximate $\mathrm{x}$ and $y$-coordinate of pupil center. Once the approximate pupil center is chosen they apply Canny edge detection [5] and Hough transformation [6] in a rectangular region centered at pupil center to detect pupil and iris boundary circle. In [78, Canny edge detection and Hough transform is used to detect pupil and iris boundary. J. M. H. Ali et al. 9] use Laplacian of Gaussian (LOG) operator for edge detection and median filter to remove the garbage pixels contain in edge of iris image. They count the black pixels in each row and column and choose the first pixel last pixel positions of the row and column which contain the maximum number of black pixels to find out pupil center and pupil radius. Mid-point algorithm of circle and ellipse are used to fit pupil boundary. Similarly, boundary fitting technique by using a coarse scale is applied to locate iris boundary. C. Tisse et al. [10] use integro-differential operators [1] with Hough transform strategy. They use gradient decomposed Hough transform to find the pupil center and iris center. In [11, a linear threshold and Freeman's chain code is used to isolate the pupillary region and then central moment is used to find 
pupil center. In [12], Canny edge detection method [5] and bisection method is used to find pupil center. Theresholding and morphological opening is used to detect pupil region and center mass of pupil region is calculated for pupil center in [13. They use active contour models (snakes) assuming the constraint that there is no internal energy to detect iris boundary. L. Liam et al. [14] converts the image to a binary image using threshold technique. They find pupil and iris by matching disk shape in the image.

\section{Proposed Approach}

We consider $\mathrm{x}$ - axis of an eye image towards vertical and $\mathrm{y}$ - axis toward horizontal as shown in Fig. 1. We use the following conventions in this paper. $r_{p}$ denotes the radius of a pupil, $x_{p}$ and $y_{p}$ denote the $x$ - and $y$-coordinate of a pupil center, respectively. $r_{i}$ denotes the radius of an iris, $x_{i}$ and $y_{i}$ denote the $x$ - and $y$ coordinate of an iris center, respectively.

In our approach, we first find pupil boundary information (namely, pupil center and pupil radius) and then use this information for detecting the iris boundary. We detect the pupil radius $\left(r_{p}\right)$ and pupil center $\left(x_{p}, y_{p}\right)$ using the steps: (i) down scaling the eye image. (ii) appling power transform and threshold the image. (iii) detecting edges. (iv) finding all connected element and removing irrelevant connected element. ( $v$ ) finding pupil center and radius using circle fitting. (vi) up scaling the pupil information to get actual values. Details of the above mentioned steps are reported in [15.

All the steps in iris boundary detection are discussed in the following sub sections.

\subsection{Preprocessing}

We propose histogram equalization and median filter as preprocessing tasks. We use pupil information and down scaled eye image in iris boundary detection step. In an eye image, low contrast between iris and sclera part is present. In this step of iris boundary detection, we use histogram equalization [16] to increase the contrast between iris and sclera part of an eye image. Histogram equalization is the technique by which the dynamic range of the histogram and contrast of an image is increased based on the intensity histogram of the image. The histogram equalization is done on an image using Eq. (11).

$$
s_{i}=\left[\sum_{j=0}^{i} n_{i}\right] \times \frac{L-1}{N}
$$

where $s_{i}$ represents the new intensity value corresponding to the $i$-th intensity level. $n_{i}$ is the number of pixel having intensity value $i, i=0,1,2, \ldots L-1$. $N$ and $L-1$ represent the total number of pixel in the image and maximum intensity level, respectively. 
We use median filter prior to the edge detection. Median filter removes the noise due to histogram equalization. Median filtering replaces pixels with the median value in the neighborhood. Figure 2 (c) shows the eye image after applying median filter of size $5 \times 5$ on the histogram equalized image.

\subsection{Edge Detection}

We use the Canny edge detection [5] method in our approach. The Canny operator works in a multi-stage process. In its first stage, we smooth the input image using Gaussian smooth operator. Gaussian smoothing reduces the noise in the eye image and also eliminates the chance of producing an edge due to noise. In the next stage, we apply Canny edge detection [5] for both horizontal and vertical edges on smoothed image. In the final stage, we suppress the local maxima. Figure 3 (a) shows the image after the Canny edge detection.

\subsection{Removing Unnecessary Edge Information}

It is observed that there is a possibility of staying many unnecessary edges present after the above mentioned steps. This is also evident in Fig 3(a). Unnecessary edges appear due to pupillary boundary, eyelids, eye lashes, non uniform illumination and blood vessel in the iris. We focus our interest only on the iris boundary edges. We try to remove above irrelevant edges as much as possible. The removal of irrelevant edges done in the following steps. We represent the pixel value by 1 for edge and 0 for no edge in an edge image.

We find out the edges caused by pupil boundary. To find the pupil boundary we use the knowledge of pupil radius $\left(r_{p}\right)$ and pupil center $\left(x_{p}, y_{p}\right)$. We remove edge pixels whose distance from pupil center $\left(x_{p}, y_{p}\right)$ is less than the pupil radius $\left(r_{p}\right)$. Mainly vertical edges form the iris boundary in the edge image. Horizontal edges occurs mainly for the eyelids of the eye images. We remove the horizontal edges of length greater than two pixel. We remove these edges following Eq. (2).

$$
O(x, y)=\left\{\begin{array}{lc}
I(x, y) \oplus(I(x, y-1) \bullet I(x, y) \bullet I(x, y+1)) & \text { if } 0 \leq x \leq N-1 \\
& 1 \leq y \leq M-2 \\
0 & y=1 \text { or } 0 ; \text { or } \\
d_{p} \leq r_{p}+20
\end{array}\right.
$$

Where, $I(x, y)$ and $O(x, y)$ represent the pixel value in edge image and removed edge image at $(x, y)$ position, respectively. $M$ and $N$ are the image width and

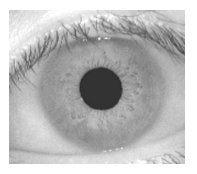

(a) Original eye image

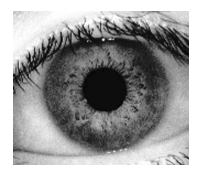

(b) Eye image after histogram

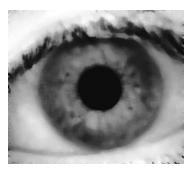

(c) Eye image after
median filter

Fig. 2. Eye image in different preprocessing step 
image height, respectively. $d_{p}$ is the distance of a pixel at $(x, y)$ from pupil center $\left(x_{p}, y_{p}\right)$ i.e. $d_{p}=\sqrt{\left(x-x_{p}\right)^{2}+\left(y-y_{p}\right)^{2}}$. $\oplus$ and $\bullet$ are two Boolean operators denoting ex-OR and AND, respectively. The value 20 is added with $r_{p}$ because from our experiments we have observed that no edges are significant as a part of iris boundary which are $r_{p}+20$ distance apart from the pupil center $\left(x_{p}, y_{p}\right)$ in all images. Figure 3(b) shows the image after removing the pupil boundary edge.

We remove the smaller edges from the image produced after horizontal edge elimination. To do this first we find out all connected components and length of the corresponding component in the image. We eliminates those connected components whose length is less than the $50 \%$ of the maximum length because edges produce at the iris and sclera region is larger than other edges. Figure 3(c) shows the edge image after removal of smaller length edges.

\subsection{Calculating Approximate Iris Radius}

Let, $\left(x_{e 1}, y_{e 1}\right),\left(x_{e 2}, y_{e 2}\right), \ldots,\left(x_{e k}, y_{e k}\right)$ be the edge points present in the edge image and $k$ be the number of edge points. We compute the distance between each edge points and pupil center in Eq. (3).

$$
d(i)=\left\lfloor\sqrt{\left(x_{e 1}-x_{p}\right)^{2}+\left(y_{e 1}-y_{p}\right)^{2}+0.5}\right\rfloor, i=1,2, \ldots, k .
$$

The number of distance levels $(L)$ computed using Eq. (4).

$$
L=\max _{i}\{d(i)\}-\min _{i}\{d(i)\}+1, i=1,2, \ldots, k .
$$

We represent each level of distance as $d_{j}, j=1,2, \ldots, L$. Now, we compute the probability of each distance occurrence in Eq. (5).

$$
p\left(d_{j}\right)=\frac{h\left(d_{j}\right)}{k}, j=1,2, \ldots, L .
$$

where, $h\left(d_{j}\right)$ is the number of edge points with distance $d_{j}$. We determine the approximate radius of iris $\left(r_{\text {approx }}\right)$ in Eq. (6).

$$
r_{\text {approx }}=\max _{d_{j}}\left\{p\left(d_{j}\right)\right\}, j=1,2, \ldots, L .
$$

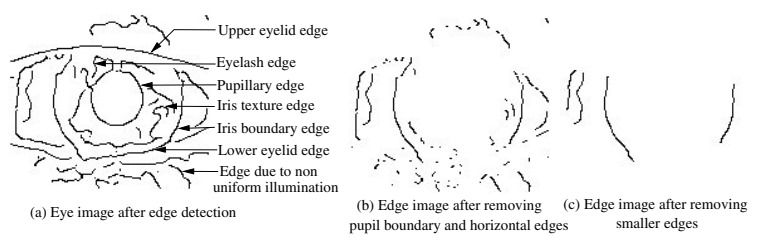

Fig. 3. Edge images at different edge removing step 
Table 1. Number of search points and search times required to find iris boundaries with and without elimination of irrelevant edges

\begin{tabular}{ccccc}
\hline Image & $\begin{array}{c}\text { With eliminating irrelevant edges } \\
\text { (our approach) }\end{array}$ & $\begin{array}{c}\text { Without Eliminating irrelevant } \\
\text { edges (existing approach) }\end{array}$ \\
\cline { 2 - 5 } & $\begin{array}{c}\text { No of search } \\
\text { points }\end{array}$ & $\begin{array}{c}\text { Search time } \\
(\mathrm{ms})\end{array}$ & $\begin{array}{c}\text { No of search } \\
\text { points }\end{array}$ & $\begin{array}{c}\text { Search time } \\
(\mathrm{ms})\end{array}$ \\
\hline Image-1 & 241 & 796.559 & 1412 & 5501.460 \\
Image-2 & 213 & 698.427 & 1306 & 4685.471 \\
Image-3 & 285 & 965.753 & 1682 & 6618.287 \\
Image-4 & 215 & 710.220 & 1318 & 4989.161 \\
Image-5 & 235 & 765.251 & 1395 & 5354.782 \\
\hline Average & 238 & 787.242 & 1422 & 5429.832 \\
\hline
\end{tabular}

\subsection{Finding Iris Center and Radius}

Finding the iris boundary is same as finding a circle from an eye image. We have already calculated the approximate radius of iris $r_{\text {approx }}$. Now, to reduce the search space we find the approximate iris center. The actual center of the circle is near to the approximation center. We choose the pupil center $\left(x_{p}, y_{p}\right)$ as approximate iris center. We consider a small region $(7 \times 7)$ around approximate circle center to find actual iris circle center. To find the actual radius of iris circle, we use all circles for radius from (approximate radius-7) to (approximate radius +7$)$ to fit circle. We consider only those points which are in the circular region with in radius $75 \%$ of $r_{\text {approx }}$ and $125 \%$ of $r_{\text {approx }}$ from center $\left(x_{p}, y_{p}\right)$. We use circle equation $\left[\left(x-x_{c}\right)^{2}+\left(y-y_{c}\right)^{2}=r_{c}^{2}\right]$ to count those points which lies on the circle for all values of $\left(x_{c}, y_{c}\right) \in(7 \times 7)$ around approximate circle center $\left(x_{p}, y_{p}\right)$ and all values of $r_{c} \in\{$ approximate radius-7,approximate radius$6, \ldots$, approximate radius +7$\}$. We choose that $\left(x_{c}, y_{c}\right)$ and $r_{c}$ for which count gives the maximum value. We also store corresponding count values as iris center $\left(x_{d n_{i}}, y_{d n_{i}}\right)$ and iris radius $\left(r_{d n_{i}}\right)$. Table 1 shows the searching time and number of pixels to be searched to fit iris boundary in scaled image for both cases: without eliminating irrelevant edges (existing approaches) and eliminating irrelevant edges (our approach). It may be noted that this iris radius and iris center are corresponding to the down sampled image.

As the processed image is in down scale (with scale factor 0.5 [15]) so we get the iris radius $\left(r_{i}\right)$ and the iris center $\left(x_{i}, y_{i}\right)$ of the original image by multiplying a factor 2 with iris radius $\left(r_{d n_{i}}\right)$ and iris center $\left(x_{d n_{i}}, y_{d n_{i}}\right)$ of down scale image.

\section{Implementation and Experimental Results}

We have implemented our approach of iris detection using C programing language in Fedora Core 5 operating system environment. We use GNU compiler GCC version 4.1.0 for compiling and executing our program. 


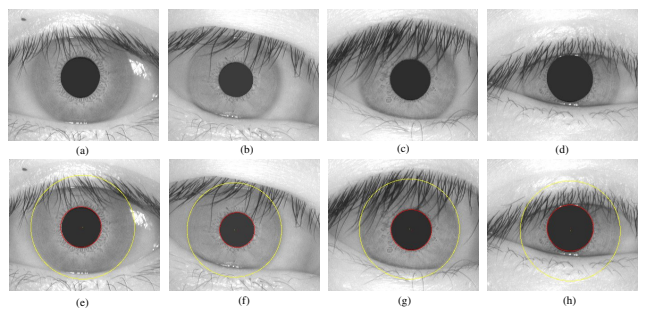

Fig. 4. Successfully detected iris boundary: (a) Eye image with light reflection, (b) Non uniform illumination eye image, (c) Eye image occluded by eyelash (d) Not properly open eye image, (e)-(h) Detected iris boundary corresponding to images in (a)-(d)

Table 2. Comparison of our work with others

\begin{tabular}{lr}
\hline Methodology & Accuracy rate \\
\hline Daugman [2] & $54.44 \%$ \\
Wildes [3] & $86.49 \%$ \\
Masek [7] & $83.92 \%$ \\
Liam and Chekima 14] & $64.64 \%$ \\
T. Mäenpä̈̈ 13] & $93 \%$ \\
This Work & $97.62 \%$ \\
\hline
\end{tabular}

Our approach has been tested on 108 different set of CASIA iris image database collected by Institute of Automation, Chinese Academy of Science [17. The database includes 756 iris images from 108 individuals. For each eye, seven images are there which have been captured in two sessions; three samples are collected from first session and four samples are collected in second session. In Fig 4(a)(d), some degraded sample images from CASIA eye image database are shown. Our approach successfully detect iris boundary though light reflection present at iris boundary (Fig. 4(a)), non uniform illumination in eye image (Fig. 4(b)), iris is occluded by eye lashes (Fig. 4(c)). This approach is also accurately find iris boundary when eye is not properly opened as shown in Fig. 4(d). Figure 4(e)-(h) show the detected iris boundary using our proposed method corresponding to the images in Fig 4 (a)-(d).

We compare our results with some best known algorithms [18. The result of comparisons is shown in Table 2 From Table 2, it is evident that proposed approach has the best performance. The accuracy rate is up to $97.62 \%$. In [812], work on iris localization have been reported. These work do not mention on accuracy rate and hence we could not compare those work with our work.

\section{Conclusions}

We propose an efficient and accurate iris detection method for developing better biometric identification system in wide spread application areas. Proposed 
approache able to detect iris boundary when eye images are not of good quality, with non-uniform illumination of images, eyes are occluded with eye lashes, only part of irises are visible etc. As a consequence, our approach is more practical than the existing approaches. Significantly our approach is $75 \%$ faster than the existing approaches and also detects iris boundary almost 98\% accurately as substantiated by experimental results on CASIA eye image database.

\section{References}

1. Daugman, J.: How iris recognition works. IEEE Trans. on Circuits and Systems for Video Technology 14(1), 21-30 (2004)

2. Daugman, J.G.: High confidence visual recognition of persons by a test of statistical independence. IEEE Trans.on PAMI 15(11), 1148-1161 (1993)

3. Wildes, R.P.: Iris Recognition: An Emerging Biometric Technology. Proc. of the IEEE 85(9), 1348-1363 (1997)

4. Ma, L., Tan, T., Wang, Y., Zhang, D.: Efficient Iris Recognition by Characterizing Key Local Variations. IEEE Trans. on Image Processing 13(6), 739-750 (2004)

5. Canny, J.: A computational approach to edge detection. IEEE Trans. on PAMI 8(6), 679-698 (1986)

6. Hough, P.: Method and means for recognizing complex patterns. U.S. Patent 3,069,654 (December 1962)

7. Masek, L.: Recognition of human iris patterns for biometric identification (2003), http://www.csse.uwa.edu.au/pk/studentprojects/libor

8. Huang, J., Wang, Y., Tan, T., Cui, J.: A New Iris Segmentation Method for Recognition. In: Proc. of the ICPR, vol. 3, pp. 554-557 (August 2004)

9. Ali, J.M.H., Hassanien, A.E.: An Iris Recognition System to Enhance E-security Environment Based on Wavelet Theory. Advanced Modeling and Optimization journal 5(2), 93-104 (2003)

10. Tisse, C.L., Martin, L., Torres, L., Robert, M.: Person identification technique using human iris recognition. In: Proc. of Vision Interface, Canada, pp. 294-299 (2002)

11. Vasta, M., Singh, R., Noore, A.: Reducing the False Rejection Rate of Iris Recognition Using Textural and Topological Fearures. Int. Jnl. of Signal Processing 2(1), 66-72 (2005)

12. Sung, H., Lim, J., Park, J., Lee, Y.: Iris Recognition Using Collarette Boundary Localization. In: Proc. of ICPR, vol. 4, pp. 857-860 (August 2004)

13. Mäenpää, T.: An Iterative Algorithm for Fast Iris Detection. In: Li, S.Z., Sun, Z., Tan, T., Pankanti, S., Chollet, G., Zhang, D. (eds.) IWBRS 2005. LNCS, vol. 3781, pp. 127-134. Springer, Heidelberg (2005)

14. Liam, L., Chekima, A., Fan, L., Dargham, J.: Iris recognition using self-organizing neural network. In: IEEE, 2002 Student Conf. on Research and Developing Systems, Malasya, pp. 169-172 (2002)

15. Dey, S., Samanta, D.: An Efficient Approach of Pupil Detection in Iris Images. Techinal Report (January 2007)

16. Ritter, G.X., Wilson, J.N.: Handbook of Computer Vision Algorithms in Image Algebra. CRC Press (1996)

17. CASIA iris image database, http://www.sinobiometrics.com

18. Proença, H., Alexandre, L.A.: UBIRIS: A noisy iris image database. In: ICIAP. vol. 1, pp. 970-977 (2005) 